\title{
Unstable anginal syndrome and pulmonary oedema due to thyrotoxicosis
}

\author{
Michael Glikson, Dov Freimark, Ron Leor, Michael Shechter, \\ Elieser Kaplinsky and Babeth Rabinowitz
}

\author{
The Heart Institute, Sheba Medical Center, Tel-Hashomer, Tel-Aviv University Sackler School of \\ Medicine, Israel
}

\begin{abstract}
Summary: We report on a 40 year old woman who presented with typical unstable angina pectoris associated with pulmonary oedema, due to poorly controlled hyperthyroidism. No cardiac abnormality was detected by echo-Doppler and nuclear ventriculography. Coronary angiography demonstrated normal coronary arteries. This case represents a new manifestation of the known association of cardiac ischaemia with hyperthyroidism in the presence of normal coronary arteries.
\end{abstract}

\section{Introduction}

Heart failure is a rare manifestation of thyrotoxicosis in otherwise healthy young women who are in sinus rhythm. ${ }^{1,2}$ Ischaemic heart disease is also uncommon in this sector of the population. ${ }^{3,4} \mathrm{~A}$ case of severe pulmonary oedema during an ischaemic episode in a 40 year old woman is reviewed herewith. This patient suffered a typical anginal syndrome, induced by poorly controlled thyrotoxicosis, in the presence of normal coronary arteries.

\section{Case report}

A 40 year old thyrotoxic woman was admitted with severe pulmonary oedema. Grave's disease was diagnosed 8 years prior to admission and the patient was treated inconsistently, though effectively, with propylthiouracil and propranolol. Three months prior to admission, her known thyrotoxic symptoms (fatigue and palpitations) worsened, and she developed typical angina pectoris on effort. Both thyrotoxic and anginal symptoms were relieved by methimazol and propranolol but recurred upon withdrawal of the treatment one month prior to her admission with increasing severity. On the day of admission she had, for the first time, a prolonged episode of anginal pain at rest lasting a few hours with severe shortness of breath.

Her past medical history was negative for any

Correspondence: Michael Glikson, M.D., The Heart Institute, Chaim Sheba Medical Center, Tel-Hashomer 52621, Israel.

Accepted: 7 June 1990 coronary risk factors, including hypertension. She had regular menses. She denied smoking or alcohol consumption, nor did she have any symptoms of intercurrent infection.

Physical examination on admission revealed severe pulmonary oedema, blood pressure of 220 / $120 \mathrm{mmHg}$ (which normalized within one hour and remained normal during the rest of hospitalization), pulse rate of 130 beats/min, temperature $36.5^{\circ} \mathrm{C}$, and large goitre with typical signs of thyrotoxicosis, which included warm, moist skin, bilateral exophthalmos with lid lag, and fine tremor. The rest of the physical examination was noncontributory. Electrocardiogram (ECG) on admission demonstrated increased voltage, sinus tachycardia with ST segment elevation of $1 \mathrm{~mm}$ in $V_{1}$ to $V_{3}$, ST depression of $2.5 \mathrm{~mm}$ in $V_{4}$ to $V_{6}$, and minimal ST depression in inferior wall leads. Chest $\mathrm{X}$-ray revealed pulmonary oedema with normal heart size. Heart muscle enzymes on admission were normal, including creatine kinase, aspartate aminotransferase and lactic dehydrogenase. Haemoglobin was $12 \mathrm{~g} / \mathrm{dl}$, glucose $6.6 \mathrm{mmol} / \mathrm{l}$, cholesterol $3.9 \mathrm{mmol} / 1$. Thyroid function tests on admission were: thyroxine (T4) $-25.6 \mu \mathrm{g} / \mathrm{dl}$ (normal range 4.7-12.0), triiodothyronine (T3) resin uptake $63 \%$ (normal range 40-55), free T4 index 32 (normal range 6-12). An echocardiogram 6 hours after admission was normal with hyperkinetic left ventricle without regions of dysfunction. No ventricular hypertrophy or valvular abnormality was detected by echo and Doppler studies. Normal flow through mitral valve suggested normal left ventricular compliance, without restriction to flow. Radionuclide ventriculography at rest demonstrated no regional wall motion 
abnormalities and the left ventricular ejection fraction was 0.60 . The patient was ventilated and treated with intravenous frusemide, morphine, isosorbide dinitrate and sublingual nifedipine. Within 8 hours the patient improved markedly without any further symptoms of heart failure or angina. She was treated with propranolol 120 $\mathrm{mg} /$ day, dexamethasone $8 \mathrm{mg} /$ day, propylthiouracil $600 \mathrm{mg} /$ day, and potassium iodide solution, with rapid resolution of thyrotoxic symptoms and signs. On the following day, the patient was totally asymptomatic, and her ECG demonstrated $T$ wave inversion in $L_{1}, V_{1}$ to $V_{3}$ and $S T$ segment elevation in $\mathrm{L}_{3}$ and AVF (Figure $1 \mathrm{~b}$ ). These changes resolved gradually during the following month. Repeated muscle enzyme determinations during first 5 days of admission did not show any rise.

Coronary angiography, performed on the 4th day after admission, demonstrated normal coronary arteries. Ventriculogram was not carried out a

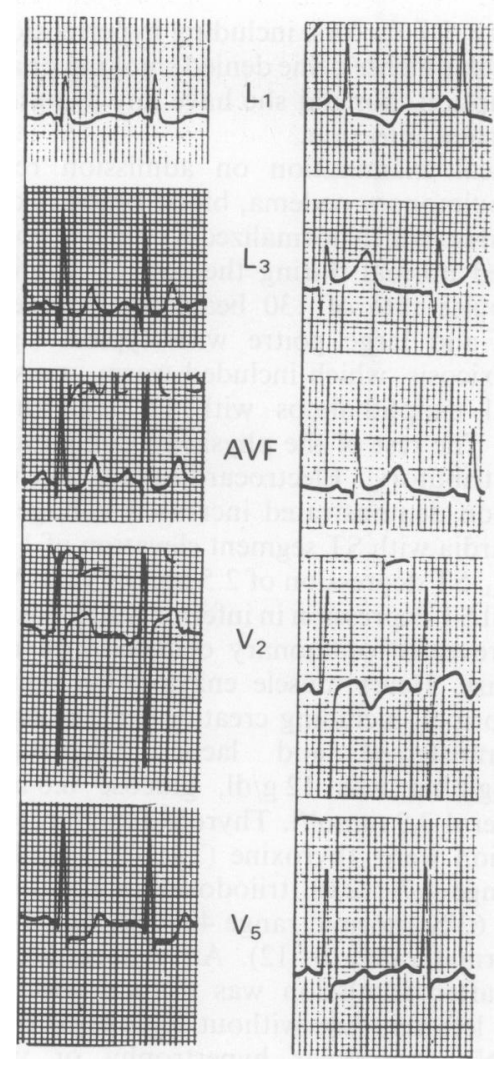

Figure 1 Electrocardiograms. (a) ECG on admission. Note ST elevation in $V_{2}$, ST depression in $V_{5}$ and minimal ST depression in $L_{3}$ and aVF. (b) ECG 24 hours after admission. Note $T$ wave inversion in $L_{1}, V_{2}$ and $S T$ elevation in $\mathrm{L}_{3}, \mathrm{aVF}$. due to normal echo-Doppler and nuclear ventriculogram. The patient was discharged on oral propranolol and propylthiouracil. On follow-up one month later, she was free of both thyrotoxic and anginal symptoms and her ECG was normal. Her thyroid function tests were normal. She underwent thyroid ablation with radioactive iodine, after which she became euthyroid without any further treatment, and has not had anginal symptoms in the following 2 years.

\section{Discussion}

The ischaemic origin of the pulmonary oedema in this patient, in spite of the presence of normal coronary arteries, is supported by the typical anginal syndrome preceding the attack of pulmonary oedema, and the sequential ECG changes. Following the classical acute ischaemic ST changes, the patient developed delayed significant negative $T$ waves, indicative of some residual damage involving the anterior wall. Although ST segment as well as $T$ wave changes are not uncommon in thyrotoxic young women, they are usually transient and much less impressive than in our case. ${ }^{5,6}$ The increased voltage is known as one of the ECG manifestation of thyrotoxicosis. ${ }^{6}$

Acute ischaemia is a well known trigger of acute transient left heart failure in patients with an otherwise adequate heart function. On the other hand, heart failure due to thyrotoxicosis per se usually develops gradually. It tends to occur in elderly patients, usually with atrial fibrillation and cardiomegaly, who frequently have concomitant heart disease, and it resolves slowly with antithyroid treatment. ${ }^{1,2,7,8}$ It has rarely been reported in young thyrotoxic women with otherwise normal heart, who are in sinus rhythm.,.$^{1,2,8-10}$

The sudden onset of pulmonary oedema in a patient without previous symptoms of failure, its rapid resolution overnight with normal echocardiogram and left ventricular function thereafter, also strongly suggest acute ischaemia as the trigger for the event.

Angina pectoris is not common in young thyrotoxic women ${ }^{3}$ and myocardial infarction is altogether quite rare. ${ }^{4,10}$ Preexisting atherosclerotic coronary artery disease was present in most of these cases, and was unmasked by hyperthyroidism. This combination, therefore, tends to occur most frequently in elderly male patients. ${ }^{3}$ There are, however, a few reports in the literature suggesting the existence of a different group of young thyrotoxic patients without any risk factor for atherosclerosis, who have myocardial ischaemia with normal coronary arteries. The mechanism underlying this phenomenon may be either an imbalance between myocardial oxygen supply and 
demand,${ }^{12}$ or coronary artery spasm, as was proven angiographically in two cases. ${ }^{12,13}$ The clinical manifestations in these patients included anginal syndrome,${ }^{12-14}$ myocardial infarction, ${ }^{15-17}$ arrhythmias ${ }^{14}$ or sudden death. ${ }^{10}$ We are not aware of any previous reports of pulmonary oedema as a mani-

\section{References}

1. Sandler, G. \& Wilson, G.M. The nature and prognosis of heart disease in thyrotoxicosis - a review of 150 patients treated with ${ }^{131}$ I. $O J$ Med 1959, 28: 347-369.

2. Griswold, D. \& Keating, J.H. Cardiac dysfunction in hyperthyroidism - a study in 810 patients. Am Heart J 1949, 38: 813-822.

3. Somerville, W. \& Levine, S.A. Angina pectoris and thyrotoxicosis. Br Heart J 1950, 12: 245-257.

4. Littman, D.S., Jeffers, W.A. \& Rose, E. The infrequency of myocardial infarction in patients with thyrotoxicosis. $\mathbf{A m ~} \mathbf{J}$ Med Sci 1957, 233: 10-15.

5. Hoffman, I. \& Lowrey, R.D. The electrocardiogram in thyrotoxicosis. Am J Cardiol 1960, 8: 893-904.

6. Sandler, G. The effect of thyrotoxicosis on the electrocardiogram. Br Heart $J$ 1959, 21: 111-116.

7. Likoff, W.B. \& Levine, S.A. Thyrotoxicosis as the sole cause of heart failure. Am J Med Sci 1943, 206: 425-434.

8. Summers, V.K. \& Surtees, S.J. Thyrotoxicosis and heart disease. Acta Med Scand 1961, 169: 661-671.

9. Schlesinger, P. \& Benchimol, A.B. The pure form of thyrotoxic heart disease. Am J Cardiol 1958, 2: 430-440.

10. Magner, C.A., Clark, W. \& Allenby, P. Congestive heart failure and sudden death in a young woman with thyrotoxicosis. West J Med 1988, 149: 86-91. festation of acute ischaemia in such a setting.

We believe that our patient had an ischaemic event terminating in severe pulmonary oedema, accounted for by the previously described association of thyrotoxicosis and ischaemia in the presence of normal coronary arteries.

11. Cheah, J.S., Lee, G.S. \& Chew, L.S. Myocardial infarction in thyrotoxicosis. Med J Aust 1971, 1: 393-395.

12. Resenkov, L. \& Falicov, R.E. Thyrotoxicosis and lactate producing angina pectoris with normal coronary arteries. $\mathrm{Br}$ Heart $J$ 1977, 39: 1051-1057.

13. Featherstone, H.J. \& Stewart, D.K. Angina in thyrotoxicosis. Arch Int Med 1983, 143: 554-555.

14. Wei, J.Y., Genecin, A., Greene, H.L. \& Achuff, S.C. Coronary spasm with ventricular fibrillation during thyrotoxicosis: response to attaining euthyroid state. $\mathrm{Am} J$ Cardiol 1979, 43: 335-339.

15. Proskey, A.J., Saksena, F. \& Towne, W.D. Myocardial infarction associated with thyrotoxicosis. Chest 1977, 72: 109-111.

16. Kotler, M.N., Michaelides, K.M., Bouchard, R.J. \& Warbasse, J.R. Myocardial infarction associated with thyrotoxicosis. Arch Int Med 1973, 132: 723-728.

17. Bergeron, G.A., Goldsmith, R. \& Schiller, N.B. Myocardial infarction, severe reversible ischemia and shock following excess thyroid administration in a woman with normal coronary arteries. Arch Intern Med 1988, 148: 1450-1452. 